\title{
Effects of Propeller-balance on Sensors in Small-scale Unmanned Aerial Vehicle
}

\author{
Masahiko Mizui ${ }^{1}$, Ikuo Yamamoto ${ }^{2}$, Ryouga Ohsawa ${ }^{2}$ \\ ${ }^{I}$ Kyushu Kyouritu University, Common Education Center \\ ${ }^{2}$ The University of Kitakyushu, Dept. of Mechanical Systems Engineering
}

\begin{abstract}
The authors are developing small UAV and studying flight stabilization control. To understand the characteristics of the aircraft is important for the control. The Vibration from rotor part has an influence on sensors which measures flight parameters. One of the reasons is the unbalance of the weight on the propeller blade. In this paper, trade edition and nylon propellers are selected for multi-rotor UAV. The static balance of a propeller can be inspected by a tool "propeller balancer." It is important to make adjustment for propellers balance at wings weight. In this paper, effect by propeller balance is verified.(current of Motor, characteristic of vibration) Centrifugal force which arises from unbalance of weight is proportional to the square of number of rotations. It was found that Resonant with Natural Frequency of arm part and vibration of rotor part was a problem to the flight-stability of UAV.
\end{abstract}

Keywords: -UAV, flight-stability, Propeller-balance, Resonant, Natural Frequency

\section{INTRODUCTION}

The study of Aerial-photography by Unmanned Aerial Vehicle (UAV). Becomes more and more important especially for disaster countermeasures. The UAV is possible to be controlled remotely by radio. Flight control using dynamics model [1] of the helicopter, it is possible to optimization of attitude on control [2] and autonomous cruise by microcontroller and sensors which measure flight parameters. "Multi-rotor" aircraft to fly more than one rotor. The rotor part composition of motors, propellers, etc. The authors are developed smallscale quadrotor [3] UAV shown in Fig.1. The author's UAV at attitude control [4] are using the PD control and the detection of air-frame angular velocity. During flight experiment at low control gain case was found a few times, the UAV with plural rotors suddenly overturn from stable state. The authors are suspicious that problem is caused by sensor malfunction. Vibration from rotor is transmitted to sensors through arm part. The first reason cause is rotor vibration by propeller balance. The vibration of high speed rotating shaft on nonlinear phenomena [5] has a significant impact on the aircraft. It thinks that resonant with natural frequency of arm part and operating variation to rotor revolutions. This resonant has an influence on sensors which measures flight parameters. This paper is investigated to characteristic of propellers. Therefore, the authors develop experimental device that reproduces to situation of single rotor. This experimental device measures thrust of propeller, number of revolutions, current of motor, sensors value for flight parameters.
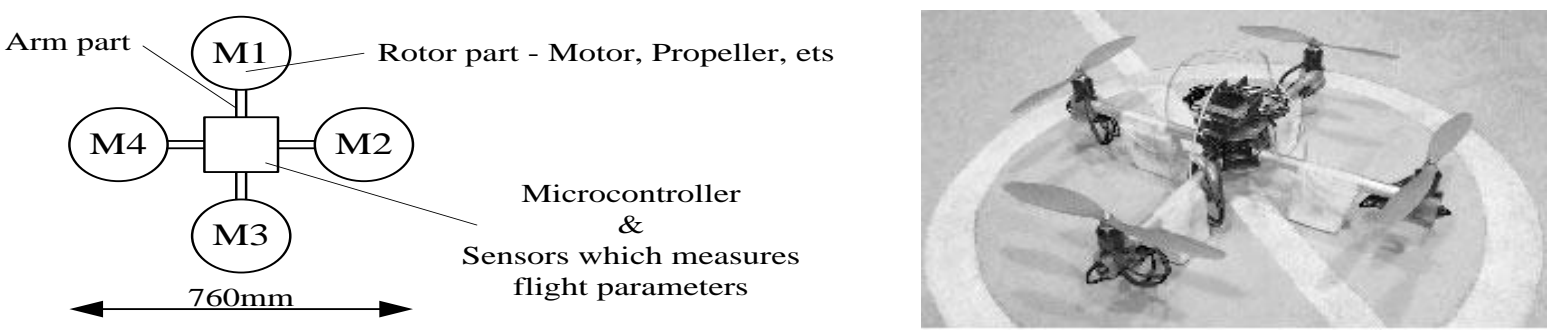

Fig.1 Small-scale Unmanned Aerial Vehicle (Quad type)

\section{EXPERIMENTAL DEVICE}

The authors development experimental device that reproduce to situation of single rotor. etc. Experimental device is shown in Fig.2. The flexibility axis of pitch is prepared in the center of arm part. Propellers, motors, and amplifiers are arranged to the both sides ends of arm part, and both side weight is adjusted with them. Arm part moves like seesaw. Microcontroller and sensors are placed in the center of arm part. A left-side motor is controlled by microcontroller, and is active. Load cell measures the thrust of left-side propeller by leverage. The number of rotations of propeller is measured with noncontact tachometer. Ammeter is connected to motor amplifier and motor current is measured. Sensors measures gyro, acceleration, posture, etc. 


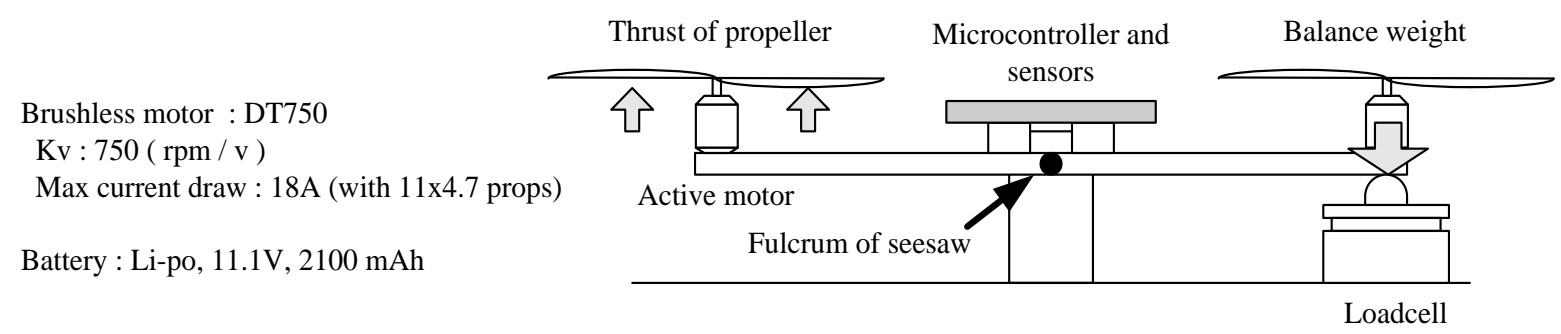

Fig.2 Experimental device

\section{PROPELLER BALANCE}

In this paper, trade edition and nylon propellers are selected for multi-rotor UAV. Trade edition propellers are shown diameter and pitch on blade. (Fig.3) The Pitch is shown forward distance at propeller single spin. Marking on propeller "11x4.7" is implied diameter: 11 inch and pitch: 4.7 inch.
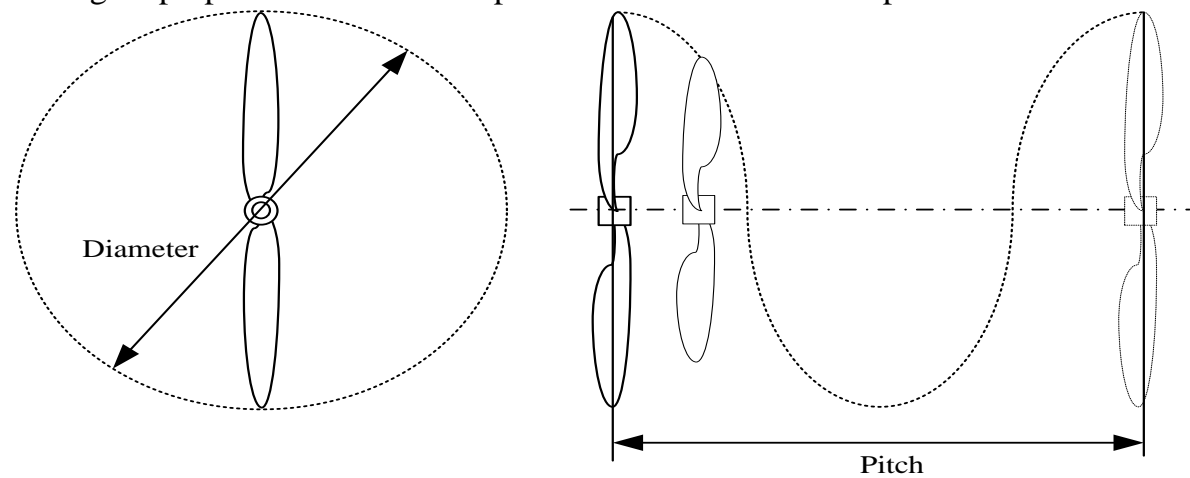

The forward distance at propeller single spin

Fig.3 Diameter and Pitch

Trade edition propellers have difference of blade weight. It is because of propeller revolution unbalance. The static balance of a propeller can be inspected by a tool "propeller balancer." Summary of propeller balancer is shown in Fig.4. Propeller balancer support propeller by magnetic float or bearings. The support spindle is reduced to resistance. The heavy side of propeller is going down with fixing the propeller balancer.

How to adjust the balance of the propeller has the following methods. a) Shave the back of the heavy side blade. b) Stick Scotch tape on the back of light side blade. c) Applying the adhesive on the back of light side blade. To adjust the balance until it converges to neutral position the propeller. In this paper, the authors propose method a) superior to durability. It is important to make adjustment for propellers balance at wings weight. It is known by fan of radio control airplane.

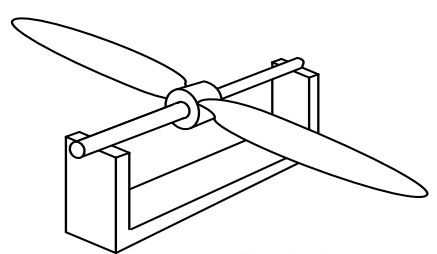

Propeller balancer

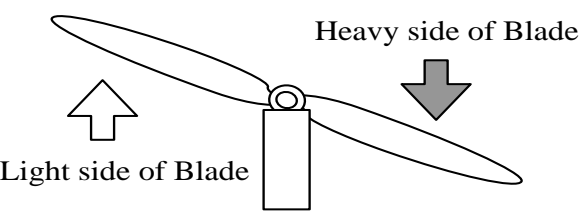

Fig.4 Propeller balancer

The vibration caused by rotational motion from unbalanced propeller will be propagated to the UAV. Unbalance of propeller is represented as centrifugal force model shown in Fig.5. Propeller balance process is reduced by the weight of unbalance "W". Mainly, the back of blade near the center is shaved. Centrifugal force is formulated from (1) and (2). Frequency is formulated from (3). Table 1 presents propeller adjustment at balance results in the case of " 10 x 4.5 " and " 11 x 4.7". To adjust shaving the back of blade center, " $r$ " is defined to $1 / 4$ of the propeller diameter " $D$ ". 

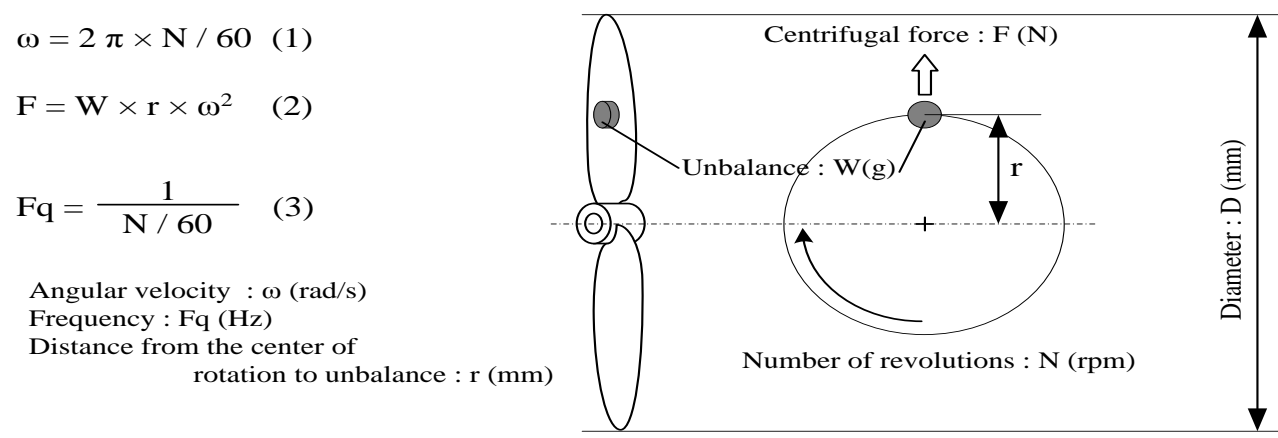

Fig.5 Centrifugal force model

Table .1 Adjustment weights of balance propellers

TYPE : $11 \times 4.7$

\begin{tabular}{|r|r|r|}
\hline $\begin{array}{l}\text { Propeller } \\
\text { weight }\end{array}$ & $\begin{array}{l}\text { Balanced } \\
\text { weight }\end{array}$ & Difference \\
\hline 10.154 & 10.014 & 0.140 \\
\hline 10.123 & 9.959 & 0.164 \\
\hline 10.169 & 9.990 & 0.179 \\
\hline \multicolumn{2}{|c}{ Weigh (g) } \\
\hline
\end{tabular}

TYPE : $10 x 4.5$

\begin{tabular}{|r|r|r|}
\hline $\begin{array}{l}\text { Propeller } \\
\text { weight }\end{array}$ & $\begin{array}{l}\text { Balanced } \\
\text { weight }\end{array}$ & Difference \\
\hline 9.239 & 9.176 & 0.063 \\
\hline 9.227 & 9.165 & 0.062 \\
\hline 9.247 & \multicolumn{2}{|c}{ Weigh (g) } \\
\hline \multicolumn{2}{r|}{} \\
\hline \multicolumn{2}{|c}{} \\
\hline
\end{tabular}

Table .2 Centrifugal forces

TYPE : $11 \times 4.7$

\begin{tabular}{|r|r|r|}
\hline Number of revolutions & Frequency & Centrifugal force \\
\hline 3000 & 50 & 1.035 \\
\hline 6000 & 100 & 4.139 \\
\hline$(\mathrm{rpm})$ & $(\mathrm{Hz})$ & $(\mathrm{N})$ \\
\hline
\end{tabular}

The authors compare the centrifugal force "F" in 3000, $6000 \mathrm{rpm}$. Assume that "11X4.7" type: unbalance weight " $\mathrm{W}$ " is $0.15 \mathrm{~g}$, Distance from the center of rotation to unbalance " $\mathrm{r} "=69.9 \mathrm{~mm}$. Table 2 presents the calculation result of centrifugal force. Centrifugal force which arises from unbalance of weight is proportional to the square of number of rotations.

\section{EFFICIENCY OF CURRENT AND THRUST}

Centrifugal force from unbalance weight will affect the rotation of the propeller. It is considered to loss of rotational energy. Therefore, the authors investigated by balance or non-balance propeller that relationship to number of revolutions between thrust and current. The authors measured from 200rpm to 2000rpm step on type "11x4.7" (Fig.6), "10x4.5" (Fig.7).

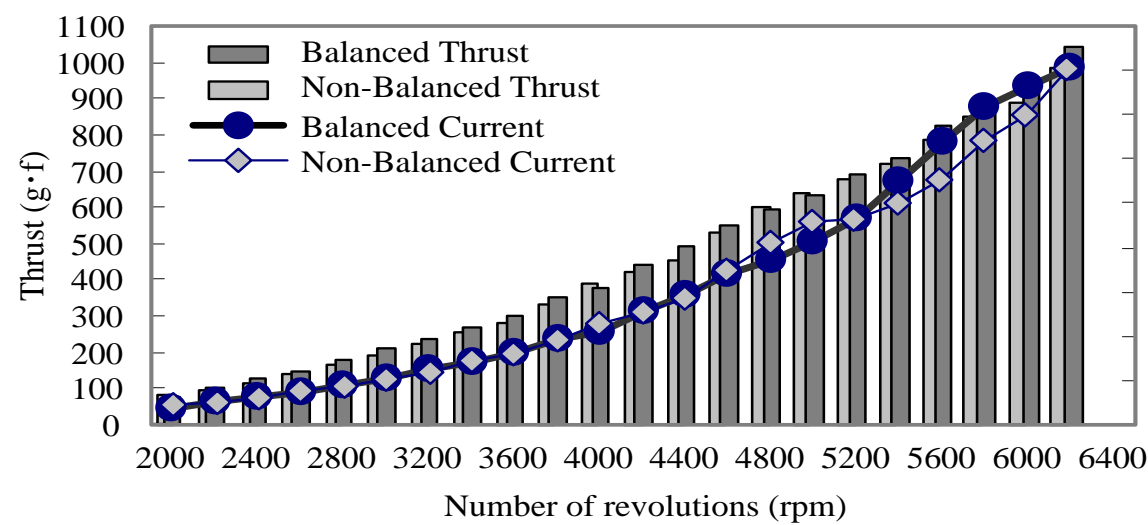

18.00

16.00

14.00

12.00

10.00

8.00

6.00

4.00

2.00

0.00

Fig.6 Type "11x4.7" Thrust and Current 


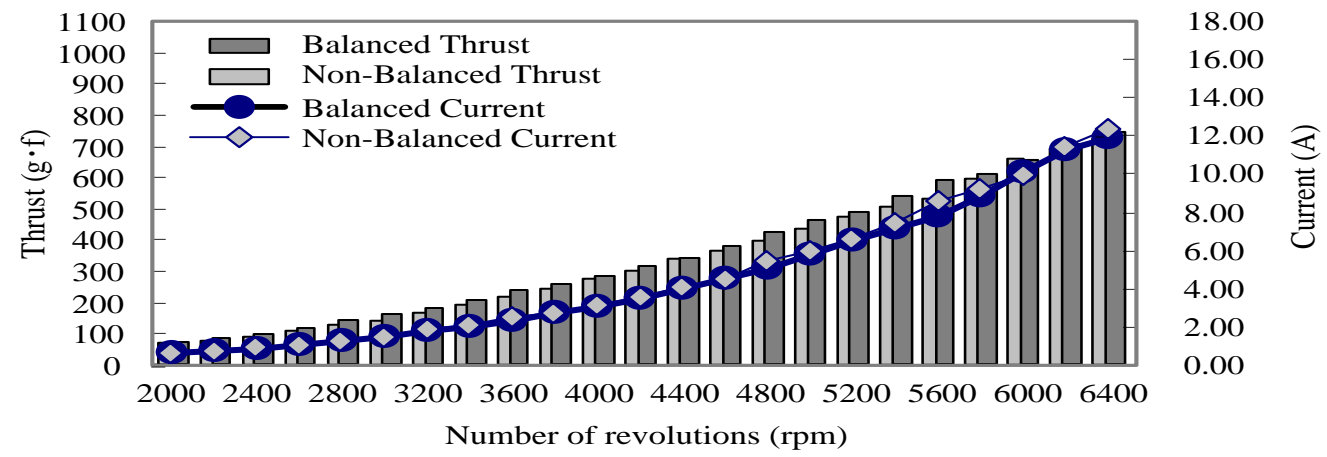

Fig.7 Type "10x4.5" Thrust and Current

Balanced propeller of "11x4.7" shows the high thrust value at high number of revolutions. The difference of the thrust value of type "10x4.5" is small. The current is increased in proportion to the number of revolutions. The influence of unbalance of weight can be considered. Balanced propeller shows a higher number of revolutions. Centrifugal force from unbalance is cause of the vibration. Then, the influence of propeller thrust. It is important to reduce the unbalance of weight.

\section{INFLUENCE FOR SENSORS WHICH MEASURES FLIGHT PARAMETERS}

Measures to unstable dynamic behavior [6], it is important to the design of the rotor. Therefore, the authors investigated by balanced or unbalanced propeller that relationship to sensors which measure flight parameters. Sensors measure cycle is period of about $10 \mathrm{~ms}$. The authors were measured to -200rpm step from 6400rpm on Type "11 $4.7 "$. Therefore, easy to measure the effect of centrifugal force is large unbalanced weight type "11x4.7". Fig.8, 9 shows the results of sensor measurements. The graph values are average ten points max and min values for each rotational speed.

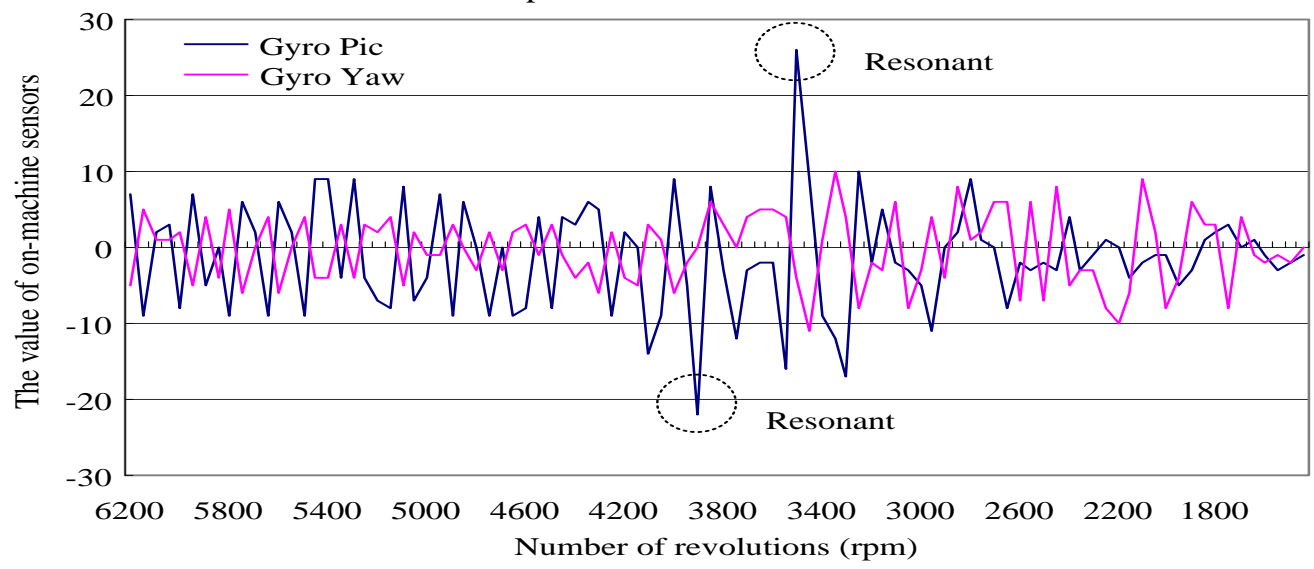

Fig.8 Sensors data in type "11x4.7" non-balanced

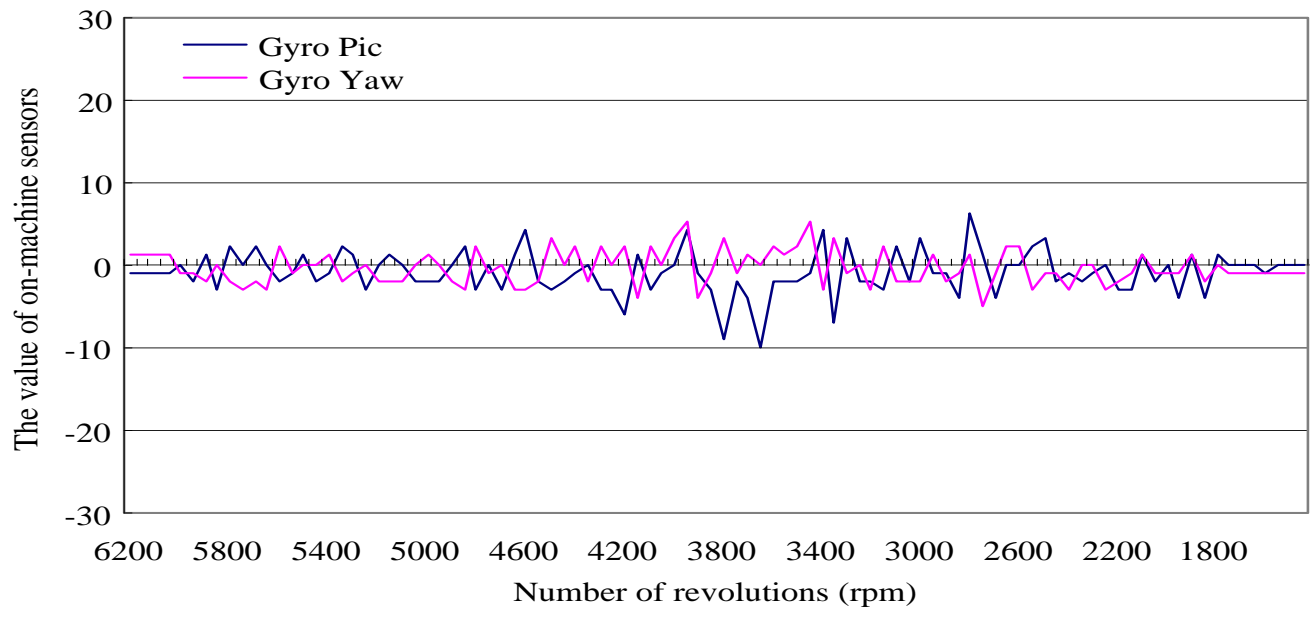

Fig.9 Sensors data in type "11x4.7" balanced 
Fig. 8 and Fig.9 graphs at $4200 \sim 3200 \mathrm{rpm}$ are also disturbed sensor values. There is a point sensor values greatly disturbed in Fig.8. At those points the rotor and the arm on the experimental device that was vibrated together. The frequency changes in proportion to the number of motor rotation. Parts of the aircraft are obtained the natural frequency from rigidity. Resonance with the vibration of rotor and the natural frequency of arm has been observed in Fig.8. Resonance is to influence the sensor value of to measure the flight parameters.

\section{CONCLUSION}

Propeller balance process is important for flying stability of the UAV. Centrifugal force which arises from unbalance of weight is proportional to the square of number of rotations. Centrifugal force from unbalance weight will affect the rotation of the propeller. It is considered to loss of rotational energy. Then, the influence of propeller thrust.

Resonance was observed by the rotor and the arm. Designed to understand the natural frequency of aircraft parts, it is important to avoid the resonance. Therefore, the designer is to understand each propellers relationship between the number of revolutions and thrust. So, there is no resonance around the number of revolutions of the rotor that thrust required for hovering.

Resonance is influenced by the sensor value of to measure the flight parameters. The sensor values from resonance will increase the amount of aircraft control. It is considered to cause overturn and hunting motion of the aircraft.

\section{REFERENCES}

[1] S.Adachi, S.Hasimoto, G.Miyamori and A.Tan, Autonomous Flight Control for a Large-Scale Unmanned Helicopter - System Identification and Robust Control Systems Design -, The transactions of the Institute of Electrical Engineers of Japan. D, A publication of Industry Applications Society 121(12), 1278-1283, 2001-12-01

[2] B.Mettler, T.Kanadev, Attitude Control Optimization for a Small-Scale Unmanned Helicopter, AIAA Guidance, Navigation, and Control Conference and Exhibit, Denver, CO, Aug. 14-17, 2000, 2000

[3] G.Hoffmann, H.Huang, S.Waslander, and C.Tomlin, Quadrotor helicopter flight dynamics and control: Theory and experiment, Proceedings of the AIAA Guidance, Navigation, and Control Conference, 2007, AIAA 2007-6461.

[4] [4] A.Tayebi,S.McGilvray, Attitude Stabilization of a VTOL Quadrotor Aircraft, IEEE Trans. on CST 14(3), 562-571, 2006

[5] Ehrich, F.F., Observations of Nonlinear Phenomena in Rotordynamics, Journal of System Design and Dynamics 2(3), 641 -651, 2008

[6] P.Corke, J.Roberts, Towards Dynamically-Favourable Quad-Rotor Aerial Robots, In Proceedings of the Australasian Conference on Robotics and Automation, Canberra, 2004 